\title{
Práticas integrativas e complementares na graduação em saúde das universidades públicas do $\mathbf{R N}$
}

\author{
Integrative and complementary practices in undergraduate health at public universities in RN \\ Prácticas integradoras y complementarias en pregrado en salud en universidades públicas en RN
}

Recebido: 21/01/2022 | Revisado: 26/01/2022 | Aceito: 31/01/2022 | Publicado: 06/02/2022

\author{
Isabel Cristina Amaral de Sousa Rosso Nelson \\ ORCID: https://orcid.org/0000-0003-4840-6950 \\ Universidade do Estado do Rio Grande do Norte, Brasil \\ E-mail: isabelrosso@uern.br \\ Janete Lima de Castro \\ ORCID: https://orcid.org/0000-0003-1823-9012 \\ Universidade Federal do Rio Grande do Norte, Brasil \\ E-mail: janetecastro.ufrn@gmail.com \\ Raiane Caroline da Silva França \\ ORCID: https://orcid.org/0000-0003-1346-1554 \\ Universidade do Estado do Rio Grande do Norte, Brasil \\ E-mail: raianecsf@gmail.com \\ Ana Tânia Lopes Sampaio \\ ORCID: https://orcid.org/0000-0003-4851-7511 \\ Universidade Federal do Rio Grande do Norte, Brasil \\ E-mail: anatsampaio@ hotmail.com
}

\begin{abstract}
Resumo
Este estudo objetiva analisar como as Práticas Integrativas e Complementares estão inseridas na graduação em saúde das Universidades públicas do RN. Foi realizada pesquisa com abordagem qualitativa, documental de caráter exploratório, na qual foram analisados 20 projetos pedagógicos de cursos de graduação em saúde e suas respectivas ementas, disponíveis publicamente nas home page dos respectivos cursos no período de agosto a novembro de 2021. Os resultados revelam pouca inserção do tema nas estruturas curriculares e, quando presente, se dão por meio de disciplinas em sua maioria optativas. Mesmo diante da necessidade da pluralidade de saberes, verifica-se pela escassez de componentes obrigatórios que abordem as práticas integrativas, a oferta em massa de componentes optativos e sua fragmentação de conteúdos, mesmo no âmbito das práticas integrativas, característico do paradigma biomédico vigente, evidenciando a necessidade de rever o ensino e formação básica desses futuros profissionais, com vistas na integralidade da atenção à saúde.
\end{abstract}

Palavras-chave: Terapias complementares; Práticas integrativas e complementares; Formação profissional em saúde; Integralidade.

\begin{abstract}
This study aims to analyze how Integrative and Complementary Practices are inserted in the health graduation course of public universities in RN. A research was carried out with a qualitative approach, documentary of an exploratory nature, in which 20 pedagogical projects of undergraduate health courses and their respective menus were analyzed, publicly available on the home pages of the respective courses from August to October 2021. The results reveal little insertion of the theme in the curricular structures and, when present, they take place through mostly optional subjects. Even in the face of the need for plurality of knowledge, there is a shortage of mandatory components that address integrative practices, the mass offer of optional components and their fragmentation of content, even within the scope of integrative practices, characteristic of the current biomedical paradigm, evidencing the need to review the basic education and training of these future professionals, with a view to comprehensive health care.

Keywords: Complementary therapies; Integrative and complementary practices; Professional training in health; Completeness.

\section{Resumen}

Este estudio tiene como objetivo analizar cómo se insertan las Prácticas Integrativas y Complementarias en la carrera de graduación en salud de las universidades públicas en RN. Se realizó una investigación con enfoque cualitativo, documental de carácter exploratorio, en la que se analizaron 20 proyectos pedagógicos de cursos de pregrado en salud y sus respectivos menús, disponibles públicamente en las páginas de inicio de los respectivos cursos de agosto a octubre de 2021. Los resultados revelan poca inserción del tema en las estructuras curriculares y, cuando están
\end{abstract}


presentes, se desarrollan a través de asignaturas mayoritariamente optativas. Incluso frente a la necesidad de pluralidad de conocimientos, hay escasez de componentes obligatorios que aborden las prácticas integradoras, la oferta masiva de componentes opcionales y su fragmentación de contenidos, incluso en el ámbito de las prácticas integradoras, característica del paradigma biomédico actual, evidenciando la necesidad de revisar la educación y formación básica de estos futuros profesionales, con miras a la atención integral de la salud.

Palabras clave: Terapias complementarias; Prácticas integradoras y complementarias; Formación profesional en salud; Lo completo.

\section{Introdução}

O Brasil vivencia uma profunda reforma no seu sistema de saúde desde 1988 com a implantação do Sistema Único de Saúde (SUS) que traz os princípios da universalidade, integralidade e equidade como basilares para mudança do modelo assistencial. Nessa perspectiva, temos um paradoxo: ao adotamos o SUS como modelo universal para nossos mais de 200 milhões de habitantes, consideramos o conceito ampliado de saúde (artigo 196 da Constituição Brasileira de 1988) que incorpora os contextos de vida da população e todo o arsenal de práticas que se orientam nesse sentido, no entanto, concomitante, observa-se que a organização dos serviços de saúde, o processo de trabalho e a formação em saúde ainda se pauta hegemonicamente pela racionalidade biomédica.

Destarte, a saúde do país vive um momento de crise que tem como raiz o próprio paradigma que rege a medicina contemporânea, segundo Luz (2005), afasta-se do sujeito humano sofredor em sua totalidade viva que deixa de ser o centro do seu objeto e do seu objetivo como prática terapêutica. A instituição da Política Nacional de Práticas Integrativas e Complementares no SUS (PNPIC) por meio da Portaria GM/MS nº 971 de 03 de maio de 2006 (Brasil, 2006) vem promover a inclusão no SUS das Práticas Integrativas e Complementares (PICS), definindo objetivos, diretrizes gerais e específicas para cada grande área de atuação das práticas.

A PNPIC propõe a estruturação e fortalecimento da atenção no SUS, em todos os níveis de atenção, com ênfase na atenção básica; em caráter multiprofissional. Propõe, ainda, o desenvolvimento de estratégias de qualificação e incentivo à divulgação e a informação dos conhecimentos básicos das PIC para profissionais de saúde, gestores e usuários, buscando parcerias que propiciem a formação para o desenvolvimento integral (Brasil, 2006).

Compreendemos que a criação da PNPIC representa um avanço no sentido da superação das contradições pontuadas anteriormente, ao incorporar algumas das práticas que constituem os itinerários terapêuticos acima descritos. O avanço da PNPIC no Brasil tem possibilitado hoje, aos usuários do SUS, o direito ao acesso a 29 PICS na rede de atenção. No que se refere às evidências científicas, as PICS tem se destacado com a instituição do Consórcio Brasileiro de Pesquisadores em Saúde Integrativa (CABSIN), com o Observatório Nacional de PICS (Observa PICS/Fiocruz/MS), com o ObservaSUS que tem uma Comunidade Virtual das PICS (Fiocruz/MS). O Ministério da Saúde e a Fiocruz têm constituído parcerias importantes com instituições acadêmicas e com os serviços oficiais de saúde em alguns estados brasileiros.

As práticas integrativas e complementares de saúde têm representado uma dimensão importante do cuidado em saúde no Brasil e no Rio Grande do Norte (RN), onde podemos evidenciar a Lei n ${ }^{\circ} 0.933$, de 17 de junho de 2021 que dispõe sobre a Política Estadual de Práticas Integrativas e Complementares (PEPIC) no âmbito da Rede de Serviços do Sistema Único de Saúde (SUS) no Estado do Rio Grande do Norte, com o rol de 32 práticas integrativas e complementares a serem ofertadas pelo SUS, que de acordo com o seu parágrafo único, devem ser incorporadas na Atenção Básica, Média e Alta Complexidades, inclusive nos Programas Nacionais de Saúde na Escola, Saúde Prisional, Saúde Mental, prioritariamente com ênfase na Atenção Básica a nas Estratégias de Atenção à Saúde da Família .

No campo da formação para o trabalho em saúde, apesar da fundamental importância, ainda há poucos estudos que apontem a inserção de conteúdos que abordem as práticas integrativas nos Projetos Pedagógicos dos Cursos (PPCs) das 
Instituições formadoras. Neste sentido, pretende-se realizar o presente estudo visando analisar como práticas integrativas e complementares estão inseridas na graduação em saúde das universidades públicas do Estado do Rio Grande do Norte.

\subsection{Contextos das PICS no RN}

No Rio Grande do Norte os serviços de PIC foram implantados timidamente no início de década de 1990, de forma isolada, por iniciativa de alguns técnicos e instituições de saúde e de alguns departamentos da Universidade Federal do Rio Grande do Norte: Saúde Coletiva; Enfermagem; Ciências Sociais, Medicina, Farmácia e Educação Física. Respaldada pela Política Nacional de Práticas Integrativas e Complementares, a Secretaria de Estado da Saúde Pública do RN, em 29 de abril de 2011, promoveu o I Seminário Estadual de Práticas Integrativas Complementares que discutiu uma proposta de Política para esta área no estado.

A proposta discutida no seminário foi aprovada na $88^{\mathrm{a}}$ reunião do Conselho Estadual de Saúde sendo então publicada no Diário Oficial do Estado-DOE a Portaria 274/ GS, de 27 de junho de 2011 que aprovou a Política Estadual de Práticas Integrativas Complementares no SUS do RN- PEPIC/RN, atendendo às diretrizes da Política Nacional de Práticas Integrativas e Complementares no SUS, do Plano Estadual de Saúde, do Plano de Diretor de Regionalização, do Plano Plurianual e da Programação Pactuada Integrada do SUS no RN.

Nesta perspectiva, foram firmadas parcerias entre Departamento de Saúde Coletiva, Núcleo de Saúde Coletiva, Departamento de Farmácia, Escola de Enfermagem da UFRN e Secretaria de Estado da Saúde Pública do RN, por meio da Coordenação de Promoção da Saúde- Grupo Estadual das PICS- SESAP, no sentido de montar um espaço que servisse de referência para os atendimentos dos usuários do SUS. Foi nesse contexto da UFRN que em 15 de maio de 2012, através do Departamento de Saúde Coletiva (DSC) e do Centro de Ciências da Saúde (CCS) foi lançado um Projeto de Extensão e uma Pesquisa-Ação que visava a "Implantação do Centro de Atenção e Pesquisa em Práticas Integrativas e Complementares CAPPIC" na UFRN, o projeto evolui consideravelmente se transformado posteriormente em um Programa de Extensão sediado Laboratório de Práticas Integrativas e Complementares em Saúde (LAPICS), criado em 30 de setembro de 2016 como uma Unidade Administrativa (15.23.01), do tipo Serviço Escola na Hierarquia organizacional da UFRN, vinculado ao Departamento de Saúde Coletiva do Centro de Ciências da Saúde. Em 28 de setembro de 2018 foi inaugurada a sede própria do LAPICS/UFRN. No município de Natal, a Política Municipal de Práticas Integrativas e Complementares em Saúde (PNPIC) foi publicada pela portaria no 135/2016-GS/SMS de 5 de maio de 2016.

Fundada em 1968, a Universidade do Estado do Rio Grande do Norte (UERN) está presente, em 06 campi distribuídos no Campus Central, em Mossoró, a segunda maior cidade do estado, além das cidades de Assú, Pau dos Ferros, Patu, Natal e Caicó, reunindo 1222 docentes e cerca de 11.600 alunos distribuídos em 59 cursos de graduação sendo destes 07 no escopo da área da saúde, 26 cursos de pós-graduação stricto sensu e 06 cursos lato sensu. Institucionalizado, no ano de 2018, o Núcleo de Práticas Integrativas e Complementares em Saúde NUPICS/UERN, se configura em núcleo de extensão vinculado a Faculdade de Enfermagem/ FAEN, que articula em seu cerne o ensino a pesquisa e a humanescência de forma efetiva, por meio dos projetos Ambulatório Integrativo, Espaço meditativo e o Pronto Socorro Energético Hospitalar.

Criada em 1967, com a ESAM - Escola Superior de Agricultura de Mossoró, e assim permanecendo até ser transformada em 29 de julho de 2005, por meio da lei $\mathrm{n}^{\circ} 11.155$ que criou a Universidade Federal Rural do Semiárido/ UFERSA. Atualmente a UFERSA está presente em 04 campi, distribuídos nas cidades de Mossoró (campus central), Angicos, Caraúbas e Pau dos Ferros, com 42 cursos de graduação, destes, apenas 02 cursos direcionados para área de saúde.

Importante referir que por meio da Portaria no 125/2019-GS/SMS de 29 de maio de 2019 foram instituídas as diretrizes da Política Municipal de Práticas Integrativas e Complementares do Sistema Único de Saúde - SUS do município de Mossoró, vinculada às áreas de Promoção da Saúde, Atenção Básica, Atenção Especializada, Saúde do Trabalhador, Gestão do 
Trabalho e Educação em Saúde. E mais recentemente, em 17 de junho de 2021, a PEPIC/RN foi sancionada como Lei Estadual de $N^{\circ} 10.931$ publicada no Diário Oficial do Estado de 18 de junho de 2021 (RN, 2021).

\subsection{Objetivo}

Analisar como as Práticas Integrativas e Complementares estão inseridas na graduação em saúde das Universidades públicas do RN.

\section{Metodologia}

Trata-se de pesquisa documental de caráter exploratório, do tipo descritiva-analítica, com abordagem qualitativa, que visa descrever como estão sendo abordadas as Práticas Integrativas e Complementares nas matrizes curriculares dos cursos de graduação em saúde das universidades públicas do Estado do Rio Grande do Norte. De acordo com Gil (2010) a análise documental é caracterizada pela pesquisa de material que irá receber uma visão analítica, de acordo com os objetos da pesquisa. Constituindo-se em uma técnica importante na pesquisa qualitativa, seja complementando informações obtidas por outras técnicas, seja desvelando aspectos novos de um tema ou problema. (Ludke \& André, 2006). A fim de reconhecer evidências das práticas integrativas e complementares em saúde no processo de formação, realizou-se análise documental dos projetos pedagógicos de curso bem como suas ementas. Nesse ínterim foram selecionados 20 cursos de graduação oferecidos por instituições públicas do Estado do Rio Grande do Norte, a saber: educação física, enfermagem, fisioterapia, fonoaudiologia, medicina, nutrição, odontologia, saúde coletiva, ordenados alfabeticamente.

Por meio desta técnica foram analisados 20 projetos pedagógicos de cursos de graduação em saúde sendo eles 13 da UFRN, 06 da UERN e 01 da UFERSA e suas respectivas ementas, disponíveis publicamente nas home pages dos respectivos cursos no período de agosto a novembro de 2021.

Este artigo resulta da pesquisa Práticas Integrativas e Complementares na Graduação em Saúde das Universidades Públicas do RN, realizada pelas autoras no âmbito do grupo de pesquisa Gestão, Educação, Trabalho e Saúde do Observatório de Recursos Humanos da UFRN, financiada pelo Fundo Nacional de Saúde mediante assinatura de Termo de Execução Descentralizada.

\section{Desenho das fases do estudo}

- Identificação dos PPCs dos Cursos disponíveis em home pages.

- $\quad$ Análise nos PPCs dos Cursos da Saúde a existência de conteúdos e/ou disciplinas em PICS

- $\quad$ Fazer uma relação entre as disciplinas oferecidas e os conteúdos da formação para o trabalho em saúde.

\section{Resultados e Discussão}

De acordo com a Constituição Federal, em seu artigo 200, compete ao SUS, entre outras atribuições, ordenar a formação dos profissionais da área de saúde. A Resolução no 569 de 8 de dezembro de 2017 em seu art. $2^{\circ}$ aprova o Parecer Técnico nº 300/2017, que apresenta princípios gerais a serem incorporados nas Diretrizes Curriculares Nacionais dos cursos de graduação da área da saúde, como elementos norteadores para o desenvolvimento dos currículos e das atividades didáticopedagógicas, e que deverão compor o perfil dos egressos desses cursos vislumbrando o profissional da saúde com formação generalista, humanista, crítica e reflexiva. Nesse ínterim todos os cursos da área de saúde definidos na Resolução MS/CNS 287 foram discutidos juntamente com o Ministério da Saúde, com vistas à adequação ao SUS.

Diante dos dispositivos legais supracitados e a mudanças de paradigmas que levaram à reformulação do ensino superior no Brasil em especial na formação do profissional de saúde, embora os avanços sejam reconhecidos, o cenário da 
educação na saúde ainda apresenta bases medicalocêntricas e verticalizadas. Mudanças na formação desses profissionais voltadas para integralidade do cuidado à saúde, formalizada como um princípio doutrinário do SUS apontam para necessidade de estratégias para a consolidação do SUS em especial, neste estudo, o ensino das práticas integrativas , em consonância com a Política Nacional de Práticas Integrativas e Complementares do SUS (Brasil, 2006).

O cenário apresentado a seguir revela que embora as PICS sejam reconhecidas no SUS com política pública de saúde estabelecida nacionalmente bem como no âmbito do Estado do Rio Grande do Norte, apenas 5\% oferta módulo específico em formato obrigatório, relacionados a temática na sua estrutura curricular, 5\% aborda conteúdo restrito de PICS em um componente de processos terapêuticos, $70 \%$ apresentam apenas componente optativo e $20 \%$ dos projetos pedagógicos avaliados, não apresentam nenhum componente relacionados à temática. Registra-se que a maioria oferta de maneira optativa, ou seja, fica a critério da oferta ou interesse do acadêmico em se aproximar com o estado da arte das PICS, conforme Gráfico 1 apresentado.

Gráfico 1 - Cenário dos PPC em relação a oferta de componentes em PICS.

\section{Cenário dos PPC em relação a oferta de componentes em pics}

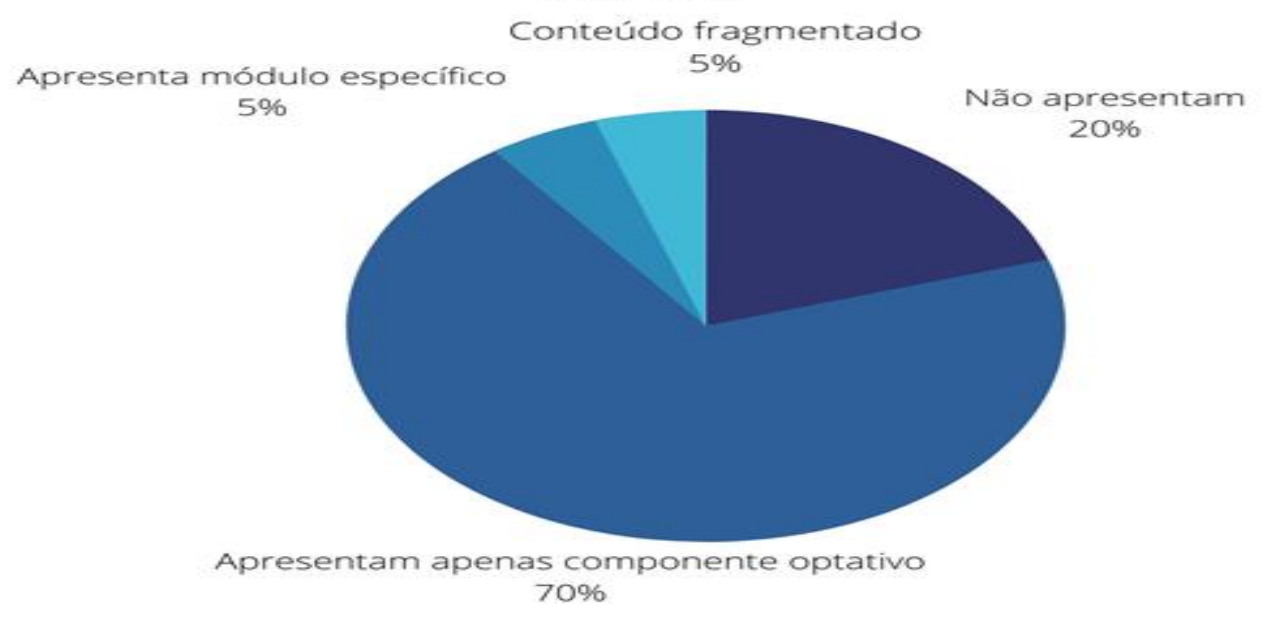

Fonte: Autores (2022).

De acordo com o Instituto Nacional de Estudos e Pesquisas Educacionais Anísio Teixeira (Inep), uma disciplina optativa é aquela que é de livre escolha para o aluno. Diante da amostra de 70\% dos cursos avaliados que só apresentam componentes e ou disciplinas optativas verificou-se que referente a carga horária, elas se apresentam com possibilidade de 30h, $45 \mathrm{~h}$ e a máxima prevista para estes é de $60 \mathrm{~h}$, conforme Gráfico 2, apresentado abaixo. 
Gráfico 2 - Carga horária dos componentes optativos.

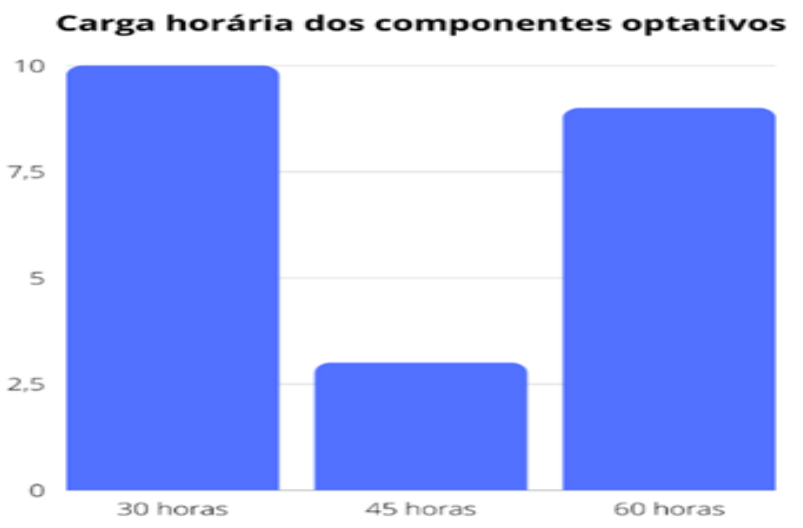

Fonte: Autores (2022).

Para melhor compreensão das ofertas dos componentes/ disciplinas optativas verificou-se as temáticas mais abordadas em seus ementários onde pode-se elencar as práticas integrativas e complementares em saúde de uma forma geral em 37,5\%, Consciência/ prática corporal em 18,8\%, Educação Popular e PICS em 18,8\%, Fundamentos da acupuntura representando 12,5\% e por fim PICS e espiritualidade também abordados em 12,5\%, conforme Gráfico 3.

Gráfico 3 - Temáticas mais abordadas nos componentes optativos.

\section{Temáticas mais abordadas nos componentes optativos}

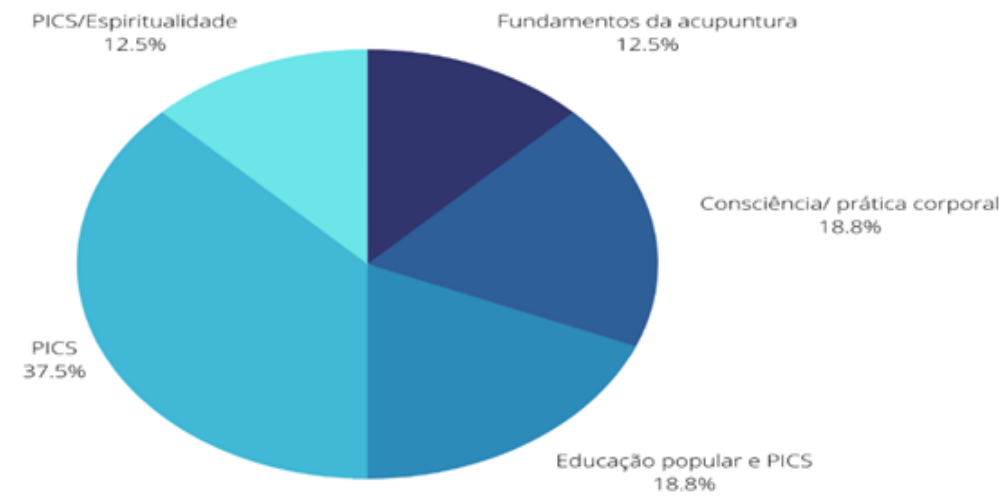

Fonte: Autores (2022).

As PICS apresentam demanda de forma exponencial onde podemos verificas como um dos maiores e principais desafios a formação profissional para sua ampliação no SUS. Uma das diretrizes da PNPIC prediz o avanço de estratégias de capacitação para profissionais que atuam com as PICS no serviço público e o incentivo à pesquisa científica e inserção de disciplinas nos cursos de graduação e pós-graduação (Brasil, 2006). Incorporar na formação em saúde o ensino de PICS requer apoios diversos em especial institucional e administrativo para incorporá-lo a um currículo já estruturado. É necessário apoio dos professores, estudantes e usuários nesse processo, além da utilização de recursos locais e incorporação de preceitos legais e culturais (Barros et al., 2011). 
Ainda existem poucos estudos sistematizados sobre o ensino em PICS no Brasil, tanto da graduação, como da pósgraduação. A maioria dos estudos foca em temas de PICS específicos em uma ou duas instituições locais, com alguns mais concentrados no campo da medicina e enfermagem, dessa forma não permite uma abordagem ampla e demonstra a necessidade de expandir pesquisas sobre este tema (Nascimento et al, 2018). A inserção das PICS nos cursos de graduação é lenta e diversificada. Além disso, é reduzido o número de cursos de saúde que ministram essa temática em suas estruturas curriculares, que são muitas vezes optativas quando oferecidos. Portanto, grande parte do conhecimento dos profissionais de saúde com formação superior vem da leitura pessoal, da vivência familiar e até mesmo da mídia e da Internet, o que mostra a vulnerabilidade da educação formal a essas práticas, e evidencia a lacuna de conhecimento do profissional de saúde, o que reduz sua área de atuação com as práticas em si, e também na orientação que deve fornecer aos seus pacientes que estão interessados em discutir e / ou utilizar tais práticas (Couto et al., 2018; Correa et al., 2018; Santos et al., 2019).

O estudo realizado por Nascimento et al. (2018) em instituições de ensino superior públicas no Estado do Rio de Janeiro, objetivou identificar a oferta de disciplinas e cursos em PICS, em nível de graduação e pós-graduação, nas 15 subáreas da saúde. A pesquisa citada constatou a oferta de ensino em PICs em 11 subáreas de saúde, com exceção apenas de Serviço Social, Nutrição e Fisioterapia. O curso de Medicina liderou essa oferta ao equivaler a 31\% do total, logo após o curso de Farmácia, com 22\%. A Enfermagem com 14\%, seguida da Terapia Ocupacional com 7\%, Educação Física, Psicologia e Saúde Coletiva (cada uma com 5\% da oferta) e Medicina Veterinária (4\%). Os cursos de Fonoaudiologia, Odontologia, Biomedicina e Ciências Biológicas, juntos, somaram 7\% da oferta total. A distribuição das temáticas em PICS nas unidades de ensino avaliadas no estudo, indica maior predominância de homeopatia, que está presente em 44,6\% das 56 instituições. Também apresenta outros temas como Práticas Corporais, Meditação, Acupuntura/MTC, Plantas Medicinais, Medicina Antroposófica entre outros (Nascimento et al, 2018).

Dentre as disciplinas identificadas no atual estudo, predominam os formatos optativos e os conteúdos informativos, o que confirma as tendências encontradas em outras pesquisas sobre o ensino de PICS. No Brasil, diversas experiências em práticas integrativas no âmbito do ensino são apresentadas principalmente de forma colaborativa como um anexo ao sistema de conhecimento em saúde (Broom \& Adams, 2013; Salles et al., 2014; Teixeira \& Lin, 2013). Diversos autores enfatizam a soberania de disciplinas optativas no ensino de PICS. Considerando que esse tipo de formação não tem sido ministrado em componentes ou disciplinas obrigatórias nos currículos da área de saúde, pode-se inferir que existe uma ampla carência de formação de egressos nessa temática. (Broom \& Adams, 2013; Salles et al., 2014; Teixeira \& Lin, 2013). A principal fragilidade identificada nesse tipo de formação fragmentada é a perda de sentido das PICS diante do modelo biomédico que mesmo que caminhe ampliando-se dentro de uma lógica integral, torna-se incapaz de integrar de fato elementos múltiplos no olhar amplo sobre a saúde (Barboni \& Carvalho, 2021).

Neste ínterim, é possível afirmar que ausência ou insuficiência da oferta de conteúdos relacionados à práticas integrativas contribui para a formação de lacunas formativas dos profissionais, e portanto interfere na capacidade de atuar com PICS com autonomia e segurança, na inserção nos serviços de saúde e na comunicação e colaboração com profissionais que atuam com diferentes processos de trabalho em saúde, como consequência, a oferta de PICS nos serviços tende a tornar-se distanciada das necessidades da população. (Nascimento et al, 2018).

A escassez de diálogo entre PICS e o ensino em saúde no âmbito do SUS contraria as instruções da Comissão Nacional de Saúde para as Diretrizes Curriculares Nacionais (DCN) no âmbito dos cursos de graduação na área da saúde. Esse documento norteia a formação voltada para atuação e defesa do SUS, além da construção de componentes curriculares que integrem os fundamentos da promoção da saúde e seus determinantes, da Educação Popular em Saúde, e das Práticas Integrativas e Complementares como elementos preponderantes para a formação, possibilitando que os futuros profissionais 
estejam aptos para reorientar os serviços de saúde; a consolidação da autonomia dos sujeitos; e a humanização e a integralidade na assistência à saúde. (Brasil, 2018).

Pesquisas revelam que os estudantes da área de saúde demonstram interesse no ensino das PICS (Azevedo \& Pelicioni, 2012, Broom \& Adams, 2013), as justificativas para tal interesse se fundamentam na contribuição para a eficácia de tratamentos, a relação interpessoal com o paciente, a integralidade do cuidado, e na diminuição de métodos invasivos e insensíveis de atenção à saúde. O conhecimento e vivência em PICS permite aos egressos ampliar suas possibilidades no cuidado em saúde, além de permitir maior liberdade de escolha terapêutica, de acordo com o perfil e necessidades de cada indivíduo/comunidade. (Tesser \& Barros, 2010).

No estudo realizado por Santiago (2020) acerca das percepções dos estudantes sobre o ensino das práticas integrativas e complementares na graduação, as respostas foram pautadas em questões como: benefícios no ensino e aprendizagem, promoção de autocuidado, geração de multiprofissionalidade e da interprofissionalidade e incentivo ao trabalho em equipe. Os profissionais que possuem formação pautada nos paradigmas que norteiam as PICS podem contribuir positivamente no relacionamento com os pacientes, reduzir abordagens insensíveis e muitas vezes invasivas, favorecendo com uma abordagem integral e tornando o trabalho em saúde mais resolutivo (Barros et al., 2011; Broom \& Adams, 2013).

Nessa perspectiva, pode-se inferir que o sucesso da ancoragem das PICS no SUS estão diretamente ligadas às possibilidades de articulação com a academia, que é responsável por fornecer profissionais no mercado de trabalho, que são capazes de reunir outros conhecimentos além dos alopáticos. Este conhecimento irá permitir que o profissional discuta as vantagens e desvantagens com aqueles pacientes que desejam usá-las, ou para aqueles que já usam, muitas vezes inadequadamente (Salles et al., 2014).

\section{Conclusão}

Ao realizar análise da abordagem das PICS na graduação em saúde das universidades públicas do RN, evidencia-se a fragilidade da temática pela frágil inserção e protagonismo entre as disciplinas ou componentes oferecidos. Nos cursos avaliados foram apresentados componentes isolados nos currículos e disciplinas predominantemente optativas. Dentre as temáticas abordadas, destaca-se Consciência/prática corporal, Educação Popular e PICS, Fundamentos da acupuntura e espiritualidade.

O déficit de conteúdos relacionados às práticas integrativas nas universidades contribui para o desenvolvimento de lacunas formativas dos egressos, interfere no processo de aprendizagem, limita a competência na realização de possibilidades terapêuticas, além de limitar a oferta de PICS nos serviços de saúde. Mesmo diante da necessidade da pluralidade de saberes e a formação generalista, verifica-se a escassez de componentes obrigatórios que abordem as PICS, a oferta em massa de componentes optativos e sua a fragmentação de conteúdos mesmo no âmbito das práticas integrativas, característico do paradigma biomédico ainda vigente, evidenciando a necessidade de rever os pilares formativos do ensino desses futuros profissionais de saúde, com vistas a integralidade da atenção e assistência. E, deste modo, promover e subsidiar renovação dos componentes curriculares na formação profissional superior em saúde, com expectativa de fortalecimento das práticas integrativas e complementares em saúde e consequentemente o fortalecimento do SUS.

Espera-se que esse estudo sirva de subsídio para pesquisas futuras e para avaliação de outros setores e processos relacionados à oferta de PICS nas universidades. Futuros estudos acerca da participação de estudantes nas atividades extensionistas que envolvem as PICS se fazem necessários, considerando que esse também se configura como um importante ambiente de formação. Pesquisas que investiguem o interesse dos alunos pelas PICS; os indicadores de matrículas nas disciplinas que abordem práticas integrativas, aprovação e evasão nessas disciplinas e o campo de atuação dos profissionais 
com PICS são fatores essenciais para enobrecer o debate a respeito da temática, ilustrar de que forma as ausências formativas têm impactado a área e promover estratégias de incentivo e fortalecimento das PICS no âmbito educacional.

\section{Referências}

Amaral, V. F. D., Cavalcante, A. S. P., Farias, Q. L. T., Ribeiro, M. A., Araújo Júnior, D. G., \& Gomes, D. F. (2018). Mobilizando estudantes em defesa do Sistema Único de Saúde (SUS): experiências interprofissionais do VER-SUS-Sobral, CE, Brasil. Interface-Comunicação, Saúde, Educação, $22,1787-1797$.

Azevedo, E. D., \& Pelicioni, M. C. F. (2011). Práticas integrativas e complementares de desafios para a educação. Trabalho, educação e saúde, 9, 361-378.

Barboni, V. G. D. A. V., \& Carvalho, Y. M. D. (2021). Práticas Integrativas e Complementares em saúde na formação em Educação Física: avanços, desafios, velhos e novos embates. Saúde e Sociedade, 30.

Barros, N. F., Siegel, P., \& Otani, M. A. (2011). O Ensino das Práticas Integrativas e Complementares: experiências e percepções. Hucitec, 171.

BRASIL. (2006). Secretaria Executiva. Secretaria de Atenção à Saúde. Departamento de Atenção Básica. Política Nacional de Práticas Integrativas e Complementares no SUS (PNPIC), Portaria n. 971, de 03 de maio de 2006. Brasília: Ministério da Saúde.

BRASIL. (2016). Conselho Nacional de Saúde. Resolução $n^{o}$ 510, de 07 de abril de 2016. Dispõe sobre as normas aplicáveis a pesquisas em Ciências Humanas e Sociais. Diário Oficial [da] República Federativa do Brasil, Brasília, DF.

BRASIL (2017). Portaria $N^{o} 849$, de 27 março de 2017. Inclui novas Práticas Integrativas e Complementares (PICS) ao SUS. Ministério da Saúde Gabinete do Ministro. http://ba.corens.portalcofen.gov.br/wpcontent/uploads/2017/03/portaria-849-27-demar\%C3\%A7o-2017-Praticasintegrativas-e-complementares2.pdf.

BRASIL. (2018). Portaria $n^{\circ}$ 702, de 21 de março de 2018. Altera a Portaria de Consolidação nº 2/GM/MS, de 28 de setembro de 2017, para incluir novas práticas na Política Nacional de Práticas Integrativas e Complementares - PNPIC.

BRASIL. (2018). Ministério da Saúde. Secretaria de Atenção à Saúde. Departamento de Atenção Básica. Manual de implantação de serviços de práticas integrativas e complementares no SUS. Ministério da Saúde, Secretaria de Atenção à Saúde, Departamento de Atenção Básica. - Brasília: Ministério da Saúde.

Broom, A., \& Adams, J. (2013). A sociologia da educação em saúde integrativa. Hucitec Editora.

Ceccim, R. B., \& Merhy, E. E. (2009). Um agir micropolítico e pedagógico intenso: a humanização entre laços e perspectivas. Interface-Comunicação, Saúde, Educação, 13, 531-542.

Ceccim, R. B. (2018). Conexões e fronteiras da interprofissionalidade: forma e formação. Interface-Comunicação, Saúde, Educação, 22, 1739-1749. https://www.scielosp.org/article/icse/2018.v22suppl2/1739-1749/

Correa, N., Soares, M. C. F., \& Muccillo-Baisch, A. L. (2018). Conhecimento do tema plantas medicinais e fitoterápicos como instrumento tecnológico na formação dos acadêmicos de enfermagem. VITTALLE-Revista de Ciências da Saúde, 30(2), 38-46.

Couto, A. G., Binz, M. C., de Moraes, A. D. J. P., Caetano, B. D. L. S., \& da Cunha, C. C. (2018). Conhecimento, uso e aceitação de acadêmicos de medicina sobre as práticas integrativas e complementares. VITTALLE-Revista de Ciências da Saúde, 30(1), 56-62.

Gil, A. C. (2002). Como elaborar projetos de pesquisa (Vol. 4, p. 175). Atlas.

Jankevicius, J. V., \& Humerez, D. C. D. (2015). Conceitos básicos das diretrizes curriculares nacionais (DCNs) dos cursos de graduação da área da saúde Internet. Brasília: Conselho Federal de Enfermagem. http://www.cofen.gov.br/conceitos-basicos-das-diretrizes-curriculares-nacionais-dcns-dos-cursosdgraduacao-da-area-de-saude_36239.html.

Nascimento, M. C. D., Romano, V. F., Chazan, A. C. S., \& Quaresma, C. H. (2018). Formação em práticas integrativas e complementares em saúde: desafios para as universidades públicas. Trabalho, Educação e Saúde, 16, 751-772.

OMS. (2013). Estratégias de la OMS sobre Medicina Tradicional 2014-2023. Hong Kong.

Paulino, F. D. L. N. (2021). Implantação da gestão de processos em um serviço de saúde do Rio Grande do Norte (Master's thesis, Universidade Federal do Rio Grande do Norte).

Rio Grande do Norte. (2021). Lei n 10.933 , de 17 de junho de 2021 que dispõe sobre a Política Estadual de Práticas Integrativas e Complementares (PEPIC) no âmbito da Rede de Serviços do Sistema Único de Saúde (SUS) no Estado do Rio Grande do Norte.

Sampaio, A. (2014). Educação em saúde: caminhos para formação integrativa. Práticas integrativas em saúde: proposições teóricas e experiências na saúde e educação. Recife: Ed. UFPE, 99-174.

Salles, L. F., Homo, R. F. B., \& da Silva, M. J. P. (2015). Práticas Integrativas e Complementares: situação do seu ensino na graduação de Enfermagem no Brasil. Revista Saúde-UNG-Ser, 8(3-4), 37-44.

Salles, L. F., Homo, R. F. B., \& da Silva, M. J. P. (2014). Situação do ensino das práticas integrativas e complementares nos cursos de graduação em enfermagem, fisioterapia e medicina. Cogitare Enfermagem, 19(4).

Santiago, G. G. C. (2020). O ensino das práticas integrativas e complementares na graduação: percepção dos estudantes. Campina Grande, Paraíba, Brasil. 
Research, Society and Development, v. 11, n. 2, e47211226041, 2022

(CC BY 4.0) | ISSN 2525-3409 | DOI: http://dx.doi.org/10.33448/rsd-v11i2.26041

Santos, G. M., Sousa Oliveira, J. M., Lima Sousa, P. V. \& dos Anjos Barros, N. V. (2019). O ensino da fitoterapia nos cursos de Nutrição em um estado do Nordeste brasileiro. RBONE-Revista Brasileira De Obesidade, Nutrição E Emagrecimento, 13(77), 68-72.

Silva, V. O, \& Santana, P. M. M. A. (2015). Curriculum content and Brazilian Health System (SUS): analytical categories, gaps and challenges. Interface: Comunicação Saúde Educação, 19(52).

Teixeira, M. Z., \& Lin, C. A. (2013). Educação médica em terapêuticas não convencionais. Revista de Medicina, 92(4), 224-235. 